\title{
Body Balance and Core Training in Parkinson's Disease: A Longitudinal Crossover Study
}

\author{
Allois Ruben 1,2, Beratto Luca1,3,4,5, Ponzano Matteo1,4, Di Leonardo Piergiacomo1, \\ Abate Daga Federico1,4,5,6, Gollin Massimiliano1,3,4,5 \\ ${ }^{1}$ Adapted Training and Performance Laboratory, University of Turin, Turin, Italy \\ ${ }^{2}$ University School of Physiotherapist, European University of Madrid, Madrid, Spain \\ ${ }^{3}$ Department of Clinical and Biological Sciences, University of Turin, Turin, Italy \\ ${ }^{4}$ Physical Capacity \& Wellness Research Center, CRAMB, ISEF, Turin, Italy \\ ${ }^{5}$ Perfomance \& Wellness Research Center, CRPB, Turin, Italy \\ ${ }^{6}$ Department of Medical Sciences, University of Turin, Turin, Italy \\ Email: matteo.ponzano@edu.unito.it
}

How to cite this paper: Ruben, A., Luca, B., Matteo, P., Piergiacomo, D.L., Federico, A.D. and Massimiliano, G. (2017) Body Balance and Core Training in Parkinson's Disease: A Longitudinal Crossover Study. Advances in Parkinson's Disease, 6, 124-130. https://doi.org/10.4236/apd.2017.64013

Received: September 21, 2017

Accepted: November 24, 2017

Published: November 27, 2017

Copyright $\odot 2017$ by authors and Scientific Research Publishing Inc. This work is licensed under the Creative Commons Attribution International License (CC BY 4.0).

http://creativecommons.org/licenses/by/4.0/

(c) (i) Open Access

\begin{abstract}
Objective: The objective of this study was to verify the effects of two different training protocols based on Core Stability and Body Balance exercises in subjects with Parkinsons's Disease. Methods: Eight elderly men with PD (age of $73 \pm 9$ years, weight $69 \pm 16 \mathrm{~kg}$, height $164 \pm 13 \mathrm{~cm}, 2 \pm 1$ of Disability Score according to Hoehn and Yahr scale). The disability score was evaluated using Hoehn and Yahr scale. This study was 12-month-long organized in 3 blocks of 4 months each. The sample was divided in two groups formed by 4 subjects each group. During the first four months, one group performed core stability exercises (CSG) while the other group exercised itself through a Balance Exercises Program (BG). After this first part of the study both protocols were stopped for the following 4 months. Finally, the group were reversed (counter balance design) for the last 4 months of physical activity. The two groups were measured twice, before (T0) and after the treatment (T1). Both treatments were designed in order to investigate the improvement in core muscles performance in order to grant a better balance control and to reduce the risk of falling. Results: statistically significant variations were found in the flight time of Sit-to-Stand $(p<0.05,+27 \%)$ and in the step length $(p<0.05,-3 \%)$. In the CSG group, statistically significant variations were detected in Speed of Steps $(p<0.05,+5 \%)$, Step Cadence $(p<0.05,+3 \%)$, and Left Stride Duration $(p<$ $0.05,-3 \%$ ) (Table 1). Conclusion: Both Body Balance and Core training can be considered two good physical exercise methods for people with PD. This study highlights the positive effects of this training protocol on legs strength improvements and on the balance control while walking.
\end{abstract}




\section{Keywords}

Parkinson's Disease, Exercises, Body Balance, Core Stability

\section{Introduction}

Parkinson's Disease (PD) is a neurodegenerative disease that consists in a progressive loss of dopaminergic neurons, involving motor symptoms such tremors, muscular rigidity, hypokiesia (that appears in the early phase of the disease) and postural instability (that may appear in a later stage) [1]. Motor disorders and posture alterations cause a strong reduction in daily life management (DLM) [2]. Parkinson's Disease can also occur at a young age, but it commonly affects elderly people [3]. These people have physical alterations such as weight loss, fatigue, reduced muscle strength, and reduced walking speed [4]. Decrements in walking speed [5] and strength [6] in elderly subjects are considered the main element of musculoskeletal fragility. The motor control alteration causes postural instability [7], increases the balance impairment and representing a serious factor of falling risk [8]. Subjects with PD report increased difficulty in maintaining balance not only during static situations, but also in dynamic one and in the transition phase between static and dynamic state during transitions between static and dynamic [9]. Gait cycle evaluations in subjects with PD [10] reported a decrease in the speed of walking and step length [11]. Yang et al. [12] showed a correlation between step length, dynamic balance and the displacement of the center of gravity. Many authors recommend physical activity to subjects suffering from $\mathrm{PD}$, including aquatic exercises [13], physical activities accompanied by music [14] and resistance training [15]. The physical decline caused by PD in its early stage can be well fought by focusing on movements able to increase muscle longevity and to maximize muscles functional capabilities [16] [17]. Therefore, the aim of this study was to verify the impact of two different physical activities schedules on muscles flexibility, spine stability, lower limbs strength, and gait cycle in subjects with PD.

\section{Materials and Methods}

\subsection{Participants and Experimental Design}

Eight elderly men with PD (age of $73 \pm 9$ years, weight $69 \pm 16 \mathrm{~kg}$, height $164 \pm$ $13 \mathrm{~cm}, 2 \pm 1$ of Disability Score) were recruited for the study. All the subjects belonged to AAPP (Associazione Amici Parkinsoniani Piemonte) a local association in the city of Turin, Italy. All the participants were volunteer and recruited through a specific advertisement. All the participants should walk without external aids to be involved in this study and all of them signed and informed consent. The disability score was evaluated using Hoehn and Yahr scale. This study was 12-month-long organized in 3 blocks of 4 months each. The sample was divided in two groups formed by 4 subjects each group. During the first four 
months, one group performed core stability exercises (CSG) while the other group exercised itself through a Balance Exercises Program (BG). After this first part of the study both protocols were stopped for the following 4 months. Finally, the group were reversed (counter balance design) for the last 4 months of physical activity. Both protocols consisted in two sessions of sixty minutes per week. The two groups were measured twice, before $\left(\mathrm{T}_{0}\right)$ and after the treatment $\left(\mathrm{T}_{1}\right)$. Both treatments were designed in order to investigate the improvement in core muscles performance in order to grant a better balance control and to reduce the risk of falling.

\subsection{Functional Evaluations}

The sit and reach test was performed as follow: the subject was seated with both legs extended and feet leaned on the sit and reach parallelepiped. The trunk flexes forward while keeping the lower limbs extended. When the maximum distance was reached, the measure was assessed through a digital distance meter installed on the parallelepiped [15]. A stabilometric platform (Tecnoboby Prokin PK 214 P, Bergamo, Italy) [15] was used to measure the following tests: 1) 60 second Bipodal Eye Test (OA); 2) 60 seconds long, closed-loop bipodic (OC) test. Every test was separated by a resting time of one minute. The analyzed variables of the Orthostatic Pressure Center (COP) were: 1) Average speed (AP): Average speed in the antero-posterior direction (mm/s); 2) Average speed (ML): mean speed in the lateral mid-direction $(\mathrm{mm} / \mathrm{s}) ; 3)$ Area $(A)$ : Surface $\left(\mathrm{mm}^{2}\right)$ described CoP; 4) Perimeter (P): path described by CoP (mm). A Spinal Mouse ${ }^{\circ}$ (Idiag, Volketswil, Switzerland) allowed to measure thoracic segment (ThSp) between the first (ThSp1) and the last thoracic vertebra (ThSp12), lumbar segment (LSp) between ThSp12 to first sacral vertebra S1, the inclination (Incl) as the slope of connection line between S1 and ThSp1. The vertebral column was evaluated in three positions: 1) Standing upright (in relaxed position, focusing on a marker at eyes level, feet aligned with shoulders, knees extended and arms along the trunk); 2) maximal flexion (legs extended, trunk bent forward); 3) maximal extension (legs extended, arms crossed over the chest, trunk extended backwards). The Sit-to-stand test was performed using an Optojump ${ }^{\circledR}$ (Microgate, Italy), in order to identify the maximum number of times the subject sits and stand in 30 seconds [15]. Finally, the gait cycle was evaluated with G-WALK (BTS S.p.A., Garbagnate Milanese, Italy, Figure 1). The participant should walk naturally in a 20 -meter shuttle path $(10+10)$. The instrument analysed the following parameters: duration, cadence, speed, stride length, \% stride length/height, gait cycle duration, step length, stance phase duration, swing phase duration, single support duration, double support duration, strides elaborated.

\subsection{Intervention Protocols}

The aim of the CSG is to increase the muscular tone of the core in the erected, 


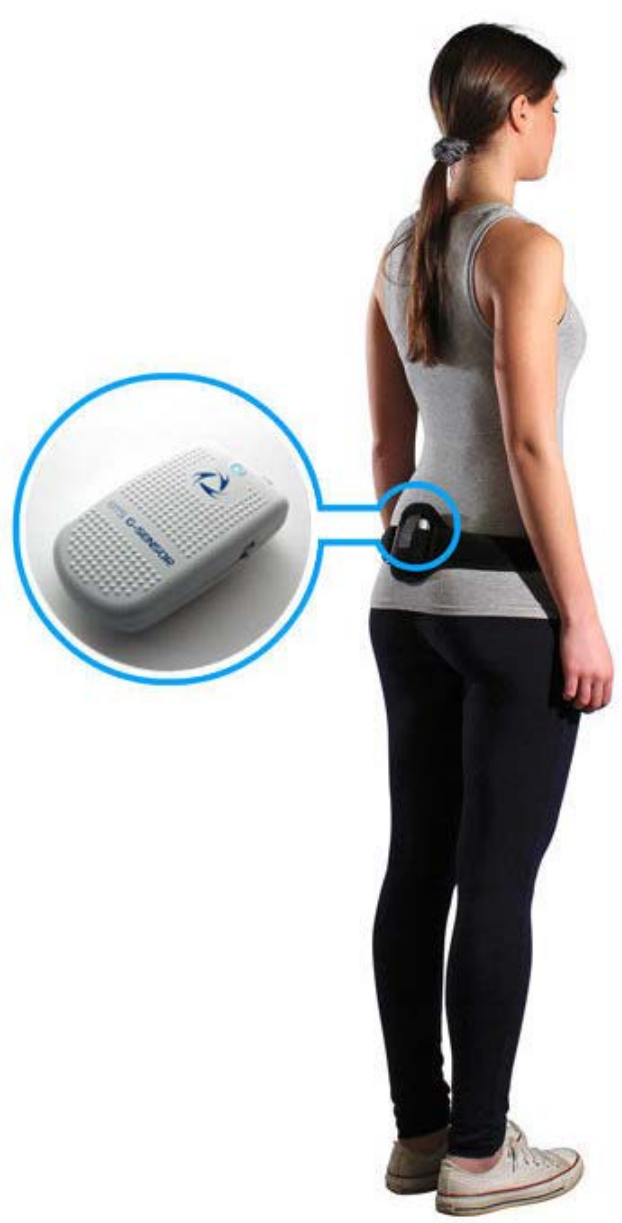

Figure 1. G-walk device and its positioning.

supine and quadruped positions (Figure 2). The aim of the BG is to improve postural stability using bi-and mono-podalic exercises on unstable surfaces (Figure 3). Each exercise includes 3 sets, $10 \pm 2$ repetitions and 1 resting minute between sets.

\subsection{Statistical Analysis}

Non parametric Wilcoxon test was used to analyze data. Percentage differences were calculated with the following formula:

$$
((F v-I v) / I v) \times 100
$$

$F_{V}$ is final value and $I_{V}$ is initial value. $p$ value was fixed at $p=0.05$.

\section{Results}

In the BG group, statistically significant variations were found in the flight time of Sit-to-Stand $(p<0.05,+27 \%)$ and in the step length $(p<0.05,-3 \%)$. In the CSG group, statistically significant variations were detected in Speed of Steps ( $p$ $<0.05,+5 \%)$, Step Cadence $(p<0.05,+3 \%)$, and Left Stride Duration $(p<0.05$, $-3 \%)$ (Table 1). 


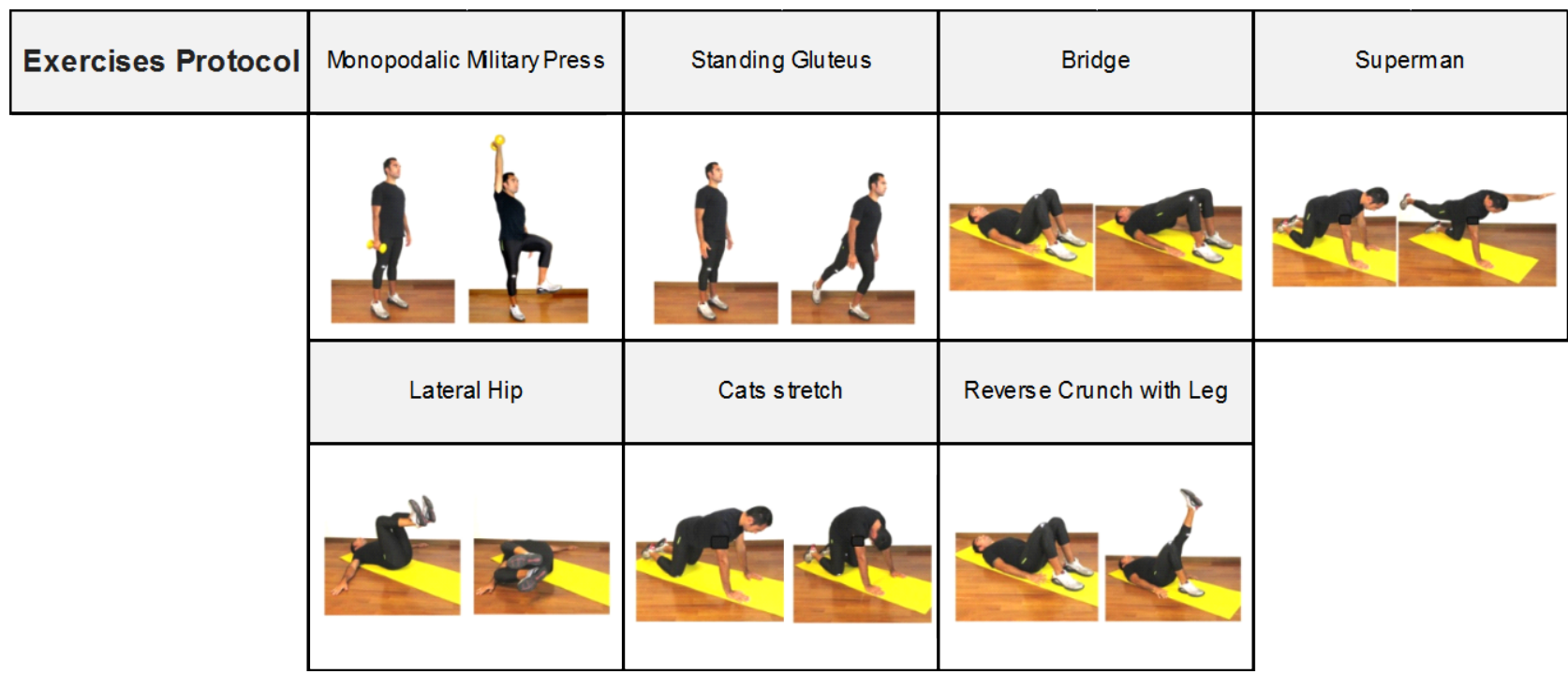

Figure 2. CSG exercises.

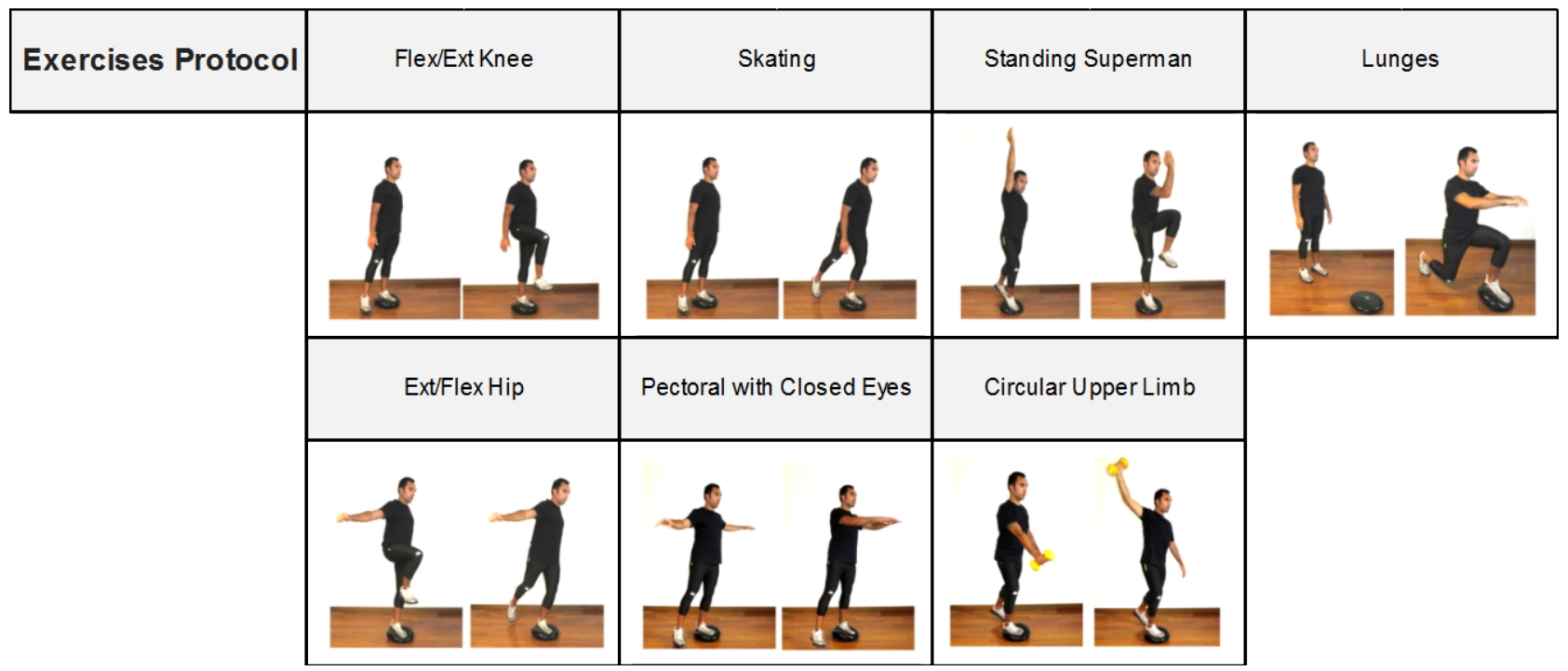

Figure 3. BG exercises.

Table 1. Results. This table highlights significant findings of both groups.

\begin{tabular}{ccccc}
\hline & Test & Variable & $p$ level & $\%$ \\
\hline \multirow{2}{*}{ BG } & Sit to Stand & Flight Time & $p<0.01$ & 27 \\
& Gait Analysis & Stride length & $p<0.05$ & -3 \\
& Gait Analysis & Speed & $p<0.05$ & 5 \\
\multirow{2}{*}{ CSG } & Gait Analysis & Cadence & $p<0.01$ & 3 \\
& Gait Analysis & Left stride duration & $p<0.01$ & -3 \\
\hline
\end{tabular}

\section{Discussion}

This study demonstrates that protocols based on core stability and balance training can be useful for people with PD. The data analysis showed a statistically 
significant improvement in the BG group in the Sit-to-Stand test. This result suggests that a training protocol based on balance control can improve the ability to manage dynamic balance and strength. In addition, the Gait Cycle analysis underlined a significant change in the stride length in both BG and CSG. This means that both methods are able to positively modify postural control during walking phase. Considering that the falling risk is enhanced by a lack of balance and postural control, we might affirm that both this training schedules are good also to reduce the falling risk in people with $\mathrm{PD}$.

\section{Conclusion}

Both Body Balance and Core training can be considered two good physical exercise methods for people with PD. This study highlights the positive effects of this training protocol on legs strength improvements and on the balance control while walking.

\section{References}

[1] Hoehn, M.M. and Yahr, M.D. (1967) Parkinsonism: Onset, Progression, and Mortality. Neurology, 17, 427-442. https://doi.org/10.1212/WNL.17.5.427

[2] Rahman, S., Griffin, H.J., Quinn, N.P. and Jahanshahi, M. (2008) Quality of Life in Parkinson's Disease: The Relative Importance of the Symptoms. Movement Disorders, 23, 1428-1434. https://doi.org/10.1002/mds.21667

[3] Crizzle, A. and Newhouse, I. (2006) Is Physical Exercise Beneficial for Persons with Parkinson's Disease? Clinical Journal of Sport Medicine, 16, 422-425. https://doi.org/10.1097/01.jsm.0000244612.55550.7d

[4] Fried, L.P., Tangen, C.M., Walston, J., Newman, A.B., Hirsch, C., Gottdiener, J., Seeman, T., Tracy, R., Kop, W.J., Burke, G. and McBurnie, M.A. (2001) Fraility in Older Adults: Evidence for a Phenotype. Journal of Gerontology: Medical Science, 56, 146-156.

[5] Shinkai, S., Watanabe, S., Kumagai, S., Fujiwara, Y., Amano, H., Yoshida, H., Ishizaki, T., Yukawa, H., Suzuki, T. and Shibata, H. (2000) Walking Speed as a Good Predictor for the Onset of Functional Dependence in a Japanese Rural Community Population. Age and Ageing, 29, 441-446. https://doi.org/10.1093/ageing/29.5.441

[6] Morley, J.E., Baumgartner, R.N., Roubenoff, R., Mayer, J. and Nair, K.S. (2001) Sarcopenia. Journal of Laboratory and Clinical Medicine, 137, 231-243. https://doi.org/10.1067/mlc.2001.113504

[7] Adkin, A.L., Frank, J.S. and Jog, M.S. (2003) Fear of Falling and Postural Control in Parkinson's Disease. Movement Disorders, 18, 496-502.

https://doi.org/10.1002/mds.10396

[8] Ashburn, A., Stack, E., Pickering, R.M. and Ward, C.D. (2001) A CommunityDwelling Sample of People with Parkinson's Disease: Charateristics of Fallers and Non Fallers. Age and Ageing, 30, 47-52. https://doi.org/10.1093/ageing/30.1.47

[9] Morris, M.E. Huxham, F., McGinley, J., Dodd, K. and Iansek, R. (2001) The Biomechanics and Motor Control of Gait in Parkinson Disease. Clinical Biomechanics, 16, 459-470. https://doi.org/10.1016/S0268-0033(01)00035-3

[10] Sofuwa, O., Nieuwboer, A., Desloovere, K., Willems, A.M., Chavret, F. and Jonkers, I. (2005) Quantitative Gait Analysis in Parkinson's Disease: Comparison with a 
Healthy Control Group. Archives of Physical Medicine and Rehabilitation, 86, 1007-1013. https://doi.org/10.1016/j.apmr.2004.08.012

[11] Morris, M., Iansek, R., Matyas, T. and Summers, J. (1998) Abnormalities in the Stride Length-Cadence Relation in Parkinsonian Gait. Movement Disorders, 13, 61-69. https://doi.org/10.1002/mds.870130115

[12] Yang, Y.-R., Lee, Y.-Y., Cheng, S.-J., Lin, P.-Y. and Wang, R.-Y. (2008) Relationships between Gait and Dynamic Balance in Early Parkinson's Disease. Gait \& Posture, 27, 611-615. https://doi.org/10.1016/j.gaitpost.2007.08.003

[13] GnoatoZotz, T.G., Souza, E.A., Israel, V.L. and Cunha Loureiro, A.P. (2013) Acquatic Physical Therapy for Parkinson's Disease. Advances in Parkinson's Disease, 2, 102-107. https://doi.org/10.4236/apd.2013.24019

[14] Hackney, M.E. and Earhart, G.M. (2009) Health-Related Quality of Life and Alternative Forms of Exercise in Parkinson Disease. Parkinsonism \& Related Disorders, 15, 644-648. https://doi.org/10.1016/j.parkreldis.2009.03.003

[15] Beratto, L., Abate Daga, F., Allois, R. and Gollin, M. (2017) Resistance Training in Parkinson's Disease: A Longitudinal Study. Advances in Parkinson's Disease, 6, 67-74. https://doi.org/10.4236/apd.2017.62007

[16] Crizzle, A. and Newhouse, I. (2006) Is Physical Exercise Beneficial for Persons with Parkinson's Disease? Clinical Journal of Sport Medicine, 16, 422-425. https://doi.org/10.1097/01.jsm.0000244612.55550.7d

[17] Musulin, I. (2017) The Effects of Exercise on People with Parkinson's Disease-Review. Advances in Parkinson's Disease, 6, 24-38. https://doi.org/10.4236/apd.2017.61003 